\title{
ACTIONS OF GROUPS OF ODD ORDER ON COMPACT, ORIENTABLE MANIFOLDS
}

\author{
CRISTIAN U. SANCHEZ
}

\begin{abstract}
Differentiable actions of cyclic groups of odd order on compact, connected, orientable manifolds with only two fixed points are studied in order to obtain conditions under which the representations of the group on the tangent spaces at the fixed points are equivalent.
\end{abstract}

A well-known theorem of Milnor establishes the equivalence of these representations in the case of a semifree action on a homology sphere.

A result for more general actions is obtained which yields a generalization of Milnor's theorem for groups of odd order.

Introduction. Let $\Theta$ be a smooth action of a cyclic group $G$, preserving the orientation of a compact, connected, orientable, even-dimensional manifold $X$ and having only two stationary points $P$ and $Q$.

Our objective is to study some conditions under which the representations $\Theta_{P}$ and $\Theta_{Q}$, of the group $G$, on the tangent spaces to $X$ at $P$ and $Q$, are equivalent.

The kind of conditions that we shall study will involve the $G$-signature of the $G$-manifold under consideration. Introduced by Atiyah, Bott, and Singer [1], [2], the $G$-signature is a powerful tool, which seems especially useful in this type of problem.

A revealing example of its power is the following theorem of Milnor which yields an important result about $h$-cobordant lens spaces [5, (12.12)].

THEOREM $([5,(12.11)],[1,(7.27)])$. Let $G$ be a compact group of diffeomorphisms of a homology sphere $X$, with fixed points $P$ and $Q$, the action being free except at $P$ and $Q$. Then the induced representations of $G$ on the tangent spaces $T_{P}$ and $T_{Q}$ are isomorphic.

Along the lines of this theorem, we study actions of a more general nature on compact orientable manifolds; that is, actions not necessarily free outside the fixed point set but with certain restrictions on the fixed point sets of the proper subgroups.

The nature of our problem restricts us to cyclic groups of odd order, which makes superfluous the initial condition of "orientation-preserving", since we

Received by the editors November 1, 1974.

AMS (MOS) subject classifications (1970). Primary 57E15, 57E25. 
shall be considering always compact, connected, orientable manifolds.

Our notation will be basically from [1] and [2], but we shall use $F(G, X)$ to indicate the fixed point set of $G$ in $X$ and, for convenience, given a point $x$ in $F(G, X), F(G, x, X)$ will indicate the connected component of $F(G, X)$ containing $x$.

The word manifold will mean smooth manifold.

I would like to express my appreciation to Professor Glen Bredon, my thesis director, who introduced me to this problem for his patience, generous advice and helpful suggestions. Special thanks also is due to Professor Ted Petrie for his teachings and many hours of enlightening conversation and to Professor Mischa Cotlar whose interest made my graduate studies possible.

1. Cyclic groups of odd order. Our first result naturally arises when one tries to obtain as much information as possible by using the $G$-signature. The idea is analogous to the one in the proof of Milnor's theorem but we use Bass' generalization of Franz' theorem.

(1.1) TheOREM. Let $G=Z_{n}$ ( $n$ odd) be a group of diffeomorphisms of a compact, connected, orientable $2 q$-manifold $X$, having only two fixed points $x$ and y. Assume that:

(a) For every proper subgroup $H$ of $G$ either $F(H, X)=\{x, y\}$ or $F(H, x, X)$ $=F(H, y, X)$.

(b) For every $g$ in $G$ satisfying $F(g, X)=\{x, y\}$, Sign $(g, X)=0$. Then $\Theta_{x}=\Theta_{y}$.

Proof. We can assume that $G$ acts as a group of isometries for a convenient metric in $X$.

Let $g$ be a generator of $G$. Since $g$ has only isolated fixed points, Sign $(g, X)=\nu(x)+\nu(y)$ and

$$
\nu(x)=\prod_{k=1}^{q}\left[\frac{e^{-i \theta_{k}}-e^{i \theta_{k}}}{\left(1-e^{-i \theta_{k}}\right)\left(1-e^{i \theta_{k}}\right)}\right]
$$

where $\theta_{k}, k=1, \ldots, q$, form a coherent system for $d g_{x}[1,(6.24)]\left(\theta_{k}\right.$ is an integral multiple of $2 \pi / n$ ).

We can assume $0<\theta_{k}<\pi$ and if we write $h_{k}=e^{-i \theta_{k}}$ then

$$
\nu(x)=\prod_{k=1}^{q}\left[\frac{1+h_{k}}{1-h_{k}}\right] .
$$

REMARK. When we take $0<\theta_{k}<\pi$, we choose an orientation on $E_{k}$ (the 2-plane direct summand of $T_{x} X$ where the action of $d g_{x}$ is rotation through $\theta_{k}$ $[1,(6.24)])$. This orientation may not agree with the original one, so we may have to multiply by $(-1)$ in the corresponding factor; this negative sign will be eliminated when we multiply by $\overline{\nu(x)}$.

Thus

$$
\nu(x) \cdot \overline{\nu(x)}=\prod_{k=1}^{q}\left[\frac{1+h_{k}}{1-h_{k}}\right] \cdot\left[\frac{1+\overline{h_{k}}}{1-\overline{h_{k}}}\right]=\prod\left[\frac{1+h_{k}}{1-h_{k}}\right]
$$


where $h_{k}$ range over all the eigenvalues of $d g_{x}$.

Now set $h=\exp (2 i \pi / n)$ and let $a_{s}^{x}$ be the number of eigenvalues of $d g_{x}$ equal to $h^{s}$; then we can write the last identity as

$$
|\nu(x)|^{2}=\prod_{s \in Z_{n} ; s \neq n}\left[\frac{1+h^{s}}{1-h^{s}}\right]^{a_{s}^{x}} .
$$

Because of our hypothesis, we have Sign $(g, X)=0$ and then $\nu(x)=-\nu(y)$, so $|\nu(x)|^{2}=|\nu(y)|^{2}$ and, therefore,

$$
1=\frac{|\nu(x)|^{2}}{|\nu(y)|^{2}}=\prod_{s \in Z_{n} ; s \neq n}\left[\frac{1+h^{s}}{1-h^{s}}\right]^{\left(a_{s}^{x}-a_{s}^{y}\right)} .
$$

Put now $a_{s}=a_{s}^{x}-a_{s}^{y}$; we have to prove $a_{s}=0$.

If $g^{\prime}$ is not a generator of $G$ but still for $H$, the subgroup generated by $g^{\prime}$, $F(H, X)=\{x, y\}$, then from $v_{g^{\prime}}(x)+v_{g^{\prime}}(y)=0$ in the same way as before we obtain

$$
\prod_{s \in Z_{n}}\left[\frac{1+h^{d s}}{e\left(h^{d s}\right)}\right]^{a_{s}}=1
$$

where $d=n /|H|$ and

$$
e(h)= \begin{cases}1-h, & h \neq 1, \\ 1, & h=1 .\end{cases}
$$

If $g^{\prime}$ generates $H$ and for this subgroup $F(H, x, X) \neq\{x\}$, then $y \in F(H, x$, $X)$ by hypothesis and therefore $\Theta_{x}\left|H=\Theta_{y}\right| H$. The expression of the coefficients of these restrictions imply that for $d=n /|H|(1.5)$ holds, so we have

$$
\prod_{s \in Z_{n}}\left[\frac{1+h^{d s}}{e\left(h^{d s}\right)}\right]^{a_{s}}=1 \quad \text { for each divisor } d \text { of } n, d \neq n .
$$

REMARK. In formulas (1.5) and (1.6) there are some factors $2^{a_{s}}$. These factors occur when $d s \equiv 0(\bmod |G|)$, that is when $s \equiv 0(\bmod |H|)$ and therefore we have in those formulas a factor $2^{t}$ with $t=\sum_{s \equiv o(|H|)} a_{s}$. Our assumptions imply that in both formulas $t=0$ and therefore this factor is just 1. In fact:

In (1.5) we are assuming that $F(H, X)=\{x, y\}$ and since $x$ and $y$ are isolated fixed points of $H$, the number of eigenvalues equal to 1 must be zero, so $\sum_{s \equiv o(|H|)} a_{s}^{x}=\sum_{s \equiv o(|H|)} a_{s}^{y}=0$ and $t=0$.

In (1.6) we are considering also the subgroups $H$ which do not satisfy $F(H, X)=\{x, y\}$, however, for these subgroups $\Theta_{x}\left|H=\Theta_{y}\right| H$ and therefore $\sum_{s \equiv o(|H|)} a_{s}^{x}=\sum_{s \equiv o(|H|)} a_{s}^{y}$ which again gives $t=0$.

We defined $a_{s}^{x}$ as the number of eigenvalues of $d g_{x}$ equal to $h^{s}$. If $p$ is an integer such that $(p, n)=1$ then $a_{s}^{x}$ is also the number of eigenvalues of $d\left(g^{p}\right)_{x}$ equal to $h^{p s}$. Thus, taking $g^{p}$ as a generator will produce formula (1.6) with $h^{p}$ instead of $h$, so this formula holds for each primitive $n$th root of unity.

Equality (1.6) can be written as 


$$
\prod_{s \in Z_{n}} e\left(h^{2 d s}\right)^{a_{s}} \cdot \prod_{s \in Z_{n}} e\left(h^{d s}\right)^{-2 a_{s}}=1 .
$$

Now $s \rightarrow 2 s$ is a bijection of $Z_{n}$ onto itself whose inverse can be denoted by $s \rightarrow s / 2$, and with this (1.7) can be written as

$$
\prod_{s \in Z_{n}} e\left(h^{d s}\right)^{\left(a_{(s / 2)}-2 a_{s}\right)}=1 .
$$

We can now apply the following theorem from [3], [4].

(1.9) Theorem (Franz-Bass). Suppose $b_{s}\left(s \in Z_{n}\right)$ are integers such that $b_{-s}=b_{s}$ and such that for each nth root of unity $h, \prod_{s \in Z_{n}} e\left(h^{s}\right)^{b_{s}}=1$. Then $b_{s}=0$ for all $s \neq n$.

The conditions of this theorem are satisfied by $b_{s}=a_{(s / 2)}-2 a_{s}$ since $a_{s}^{x}=a_{-s}^{x}$ and $a_{s}^{y}=a_{-s}^{y}$. We have therefore $a_{(s / 2)}-2 a_{s}=0$, which in turn implies $a_{s}=0$ for each $s \in Z_{n}, s \neq n$, and proves (1.1).

(1.10) COROllary. Let $X$ be a compact, connected, orientable 2q-manifold supporting an action of $G=Z_{n}$ ( $n$ odd) under the conditions of Theorem (1.1). Let $H \subset G$ be a subgroup such that $|G / H|=p^{r}$ ( $p$ prime) and $y \in F(H, x, X)$.

Consider the action of $G / H$ on $F(H, x, X)$. If $u$ is a generator of $G / H$ then $\operatorname{Sign}(u, F(H, x, X))=0$.

Proof. Since we have the equality of the representations $\Theta_{x}$ and $\Theta_{y}$, then the representations of $G / H$ on $T_{x} F(H, x, X)$ and $T_{y} F(H, x, X)$ are equivalent and so $v_{u}(x)= \pm v_{u}(y)$. Under our hypothesis, $2 v_{u}(x)$ cannot be an algebraic integer, so Sign $(u, F(H, x, X))=0$.

(1.11) COROLlaRY. Let $X$ be a rational homology sphere supporting an action of $Z_{n}$ ( $n$ odd) as a group of diffeomorphisms with only two fixed points $x$ and $y$ and satisfying $(a)$ of (1.1). Then $\Theta_{x}=\Theta_{y}$.

(1.12) Corollary. Let $Z_{s}$, podd prime, act smoothly on a $Z_{p}$-homology sphere $X$, with only two fixed points $x$ and $y$. Then $\Theta_{x}=\Theta_{y}$.

\section{REFERENCES}

1. M. F. Atiyah and R. Bott, The Lefschetz fixed point theorem for elliptic complexes. II. Applications, Ann. of Math. (2) 88 (1968), 451-491. MR 38\#731.

2. M. F. Atiyah and I. M. Singer, The index of elliptic operators. III, Ann. of Math. (2) 87 (1968), 546-604. MR 38 \#5245.

3. G. de Rham, S. Maumary and M. A. Kervaire, Torsion et type simple d'homotopie, Lecture Notes in Math., no. 48, Springer-Verlag, Berlin and New York, 1967. MR 36 \#5943.

4. H. Bass, The Dirichlet unit theorem, induced characters, and Whitehead groups of finite groups, Topology 4 (1965), 391-410. MR 33\#1341.

5. J. W. Milnor, Whitehead torsion, Bull. Amer. Math. Soc. 72 (1966), 358-426. MR 33 \#4922.

I.M.A.F. Universidad de Córdoba, Valparaiso y R. Martinez, Ciudad Universitaria, Córdoba, Argentina 